\title{
Interplay of vacancies, hydrogen, and electrical compensation in irradiated and annealed $n$-type $\beta-\mathrm{Ga}_{2} \mathrm{O}_{3}$
}

\author{
A. Karjalainen, ${ }^{1,2, a)}$ P. M. Weiser, ${ }^{3}$ I. Makkonen, ${ }^{2,4}$ V. Reinertsen, ${ }^{3}$ L. Vines, $^{3}$ and F. Tuomisto ${ }^{1,2,4}$ \\ ${ }^{1)}$ Department of Applied Physics, Aalto University, P.O. Box 15100, FI-00076 Espoo, \\ Finland \\ ${ }^{2)}$ Department of Physics, University of Helsinki, P.O. Box 43, FI-00014 Helsinki, Finland \\ ${ }^{3)}$ Department of Physics, Centre for Materials Science and Nanotechnology, University of Oslo, Blindern P.O. Box 1048, Oslo, \\ Norway \\ ${ }^{4)}$ Helsinki Institute of Physics, P.O. Box 64, FI-00014 Helsinki, Finland
}

(Dated: 3 March 2021)

Positron annihilation spectroscopy, Fourier transform-infrared absorption spectroscopy, and secondary ion mass spectrometry have been used to study the behavior of gallium vacancy-related defects and hydrogen in deuterium (D) implanted and subsequently annealed $\beta-\mathrm{Ga}_{2} \mathrm{O}_{3}$ single crystals. The data suggest the implantation generates a plethora of $V_{\mathrm{Ga}}$-related species, including $V_{\mathrm{Ga}}$ - and $V_{\mathrm{Ga} 2}$-type defects. The latter's contribution to the positron signal was enhanced after an anneal at $300^{\circ} \mathrm{C}$, which is driven by the passivation of $V_{\mathrm{Ga}}^{\mathrm{ib}}$ by hydrogen as seen from infrared measurements. Subsequent annealing near $600^{\circ} \mathrm{C}$ returns the positron signal to levels similar to those in the as-received samples, which suggests split $V_{\mathrm{Ga}}$-like defects are still present in the sample. The almost complete removal of the $V_{\mathrm{Ga}}^{\mathrm{ib}}-2 \mathrm{D}$ vibrational line, the appearance of new weak O-D lines in the same spectral region, and the lack of D out-diffusion from the samples suggest that the $600^{\circ} \mathrm{C}$ anneal promotes the formation of either D-containing, IR-inactive complexes or defect complexes between $V_{\mathrm{Ga}}^{\mathrm{ib}}-2 \mathrm{D}$ and other implantation-induced defects. The degree of electrical compensation is found to be governed by the interactions between the Ga vacancies and hydrogen.

\section{INTRODUCTION}

$\beta-\mathrm{Ga}_{2} \mathrm{O}_{3}$ is an ultrawide bandgap $(4.9 \mathrm{eV})$ semiconductor with a high theoretical electric breakdown field $\left(8 \mathrm{MV} \mathrm{cm}^{-1}\right)$, which gives it excellent potential as a successor for $\mathrm{GaN}$ and $\mathrm{SiC}$ in UV and high power electronics applications. 11 -type doping can be achieved with $\mathrm{Sn}$ or $\mathrm{Si}$ doping ${ }^{1 / 2}$ with typical carrier concentrations 1 in the range of $10^{16}-10^{19} \mathrm{~cm}^{-3}$, which has led to the development of a number of unipolar devices. ${ }^{3}$ However, an understanding of the mechanisms that lead to compensation of the $n$-type conductivity is not very well established. In particular, electrical measurements ${ }^{4}$ show that proton irradiation strongly decreases the charge carrier concentration, presumably by the formation of compensating acceptors. A compensating acceptor with an optical threshold at $\approx 2 \mathrm{eV}$ below the conduction band minimum was reported by several authors ${ }^{[5]}$ Recent investigations using steady-state photocapacitance measurements 9 tentatively assigned this signature to a Ga-related primary intrinsic defect. Indeed, theory ${ }^{4[10}$ finds that gallium vacancies $\left(V_{\mathrm{Ga}}\right)$ are deep acceptors and therefore likely candidates for these species. These observations are consistent with the results of early positron annihilation spectroscopy (PAS) experiments 11 , where a strong correlation between $V_{\mathrm{Ga}}$ and the degree of compensation of $n$-type conductivity ${ }^{12 \sqrt{13}}$ was seen. Recent studies utilizing deep-level transient spectroscopy (DLTS) have shown that irradiation also gives rise to two charge state transition levels labeled E2* and E4! $\left.{ }^{*} \mid 14\right]$ Annealing the samples at $600 \mathrm{~K}$ removes the $\mathrm{E} 4$ level but also increases the concentration of E2*! In fact, E2* has been shown limit

\footnotetext{
a)Electronic mail: antti.j.karjalainen@iki.fi
}

the performance $\beta$ - $\mathrm{Ga}_{2} \mathrm{O}_{3}$-based metal-semiconductor field effect (MESFET) transistors $\$ 17 / 18$ and suggested to originate from $V_{\mathrm{Ga}}-V_{\mathrm{O}}$ divacancy defect complexes.1619

One of the difficulties in studying intrinsic point defects in $\beta-\mathrm{Ga}_{2} \mathrm{O}_{3}$ is the large number of $V_{\mathrm{Ga}}$ configurations. Theory finds that monovacancies of both of the inequivalent Ga sites $\left(V_{\mathrm{Ga} 1}\right.$ and $\left.V_{\mathrm{Ga} 2}\right)$, are metastable, and exhibit rather low energy barriers $(<0.5 \mathrm{eV})$ to relax into "split-interstitial" (or shifted) configurations $\left(V_{\mathrm{Ga}}^{\mathrm{i}}\right)$ in $n$-type material. ${ }^{40} \mathrm{Scan}$ ning transmission electron microscopy ${ }^{20}$ has observed both these $V_{\mathrm{Ga}}^{\mathrm{i}}$ defects and their complexes with Sn dopants in as-grown single crystals. Furthermore, a higher $\mathrm{Sn}$ concentration appears to enhance the $V_{\mathrm{Ga}}^{\mathrm{i}}$ concentration, which was also suggested by theory ${ }^{4 \mid 10}$ Electron paramagnetic resonance (EPR) studies ${ }^{21} 23$ have found two defect signals labeled as EPR1 and EPR2 from which EPR1 was recently suggested to arise from $V_{\mathrm{Ga}}^{\mathrm{ib}}$ or $V_{\mathrm{Ga}}$. Recent positron annihilation studies show that $V_{\mathrm{Ga}}^{\mathrm{i}}$ can be present in high concentrations $\left(\gtrsim 1 \times 10^{18} \mathrm{~cm}^{-3}\right)$ in as-received $\beta-\mathrm{Ga}_{2} \mathrm{O}_{3}$ single crystals regardless of the growth method or doping concentration. $24 \sqrt[25]{ }$ Fourier transform-infrared (FT-IR) spectroscopy is often used to study hydrogen $(\mathrm{H})$-related defects via the absorption of localized $\mathrm{O}-\mathrm{H}$ vibrations. Although $\mathrm{H}$ and $\mathrm{D}$ generally exhibit similar physical behaviors, $\mathrm{D}$ is often used as a probe in place of $\mathrm{H}$ due to its higher sensitivity in certain techniques, e.g., FT-IR and secondary ion mass spectrometry (SIMS). A number of $\mathrm{OH}$-related defects have been reported in $\beta$ $\mathrm{Ga}_{2} \mathrm{O}_{3}$, 29 , and the dominant defect has been identified from the polarization properties of the vibrational line to be $V_{\mathrm{Ga}}^{\mathrm{ib}}$ $2 \mathrm{H} !^{26 / 27}$ The diffusion of $\mathrm{D}$ in ion-implanted $\beta-\mathrm{Ga}_{2} \mathrm{O}_{3}$ single crystals was investigated using SIMS ${ }^{30 \mid 31}$ and shown to be highly anisotropic and limited by intrinsic defects that serve as traps for D ${ }^{32}$ The traps could be present in as-received crystals, consistent with the recent positron annihilation results. 
In this work, we combined positron annihilation spectroscopy, FT-IR spectroscopy, and SIMS to study the evolution of $V_{\mathrm{Ga}}$ and $\mathrm{H} / \mathrm{D}$ in $\beta-\mathrm{Ga}_{2} \mathrm{O}_{3}$ single crystals after $\mathrm{D}$ implantation and subsequent thermal annealing. We utilize stateof-the-art theoretical calculations of positron annihilation signals for detailed analysis of the experimental data. First, it is found that irradiation creates a multitude of $V_{\mathrm{Ga}}$-related defects, including those containing $V_{\mathrm{Ga} 1}$ - and $V_{\mathrm{Ga} 2}$-type defects. Annealing at $300{ }^{\circ} \mathrm{C}$ was found to increase the irradiationgenerated defect positron signal, and also to strongly enhance the intensity of the $V_{\mathrm{Ga}}^{\mathrm{ib}}-2 \mathrm{D}$ vibrational line. Interestingly, the electrical conductivity lost in the irradiation is actually recovered in the annealing at $300^{\circ} \mathrm{C}$. Annealing at near $600^{\circ} \mathrm{C}$ returns the positron signal to the that seen in the as-received sample. The anneal also results in the complete disappearance of the $V_{\mathrm{Ga}}^{\mathrm{ib}}-2 \mathrm{D}$ line and the appearance of new O-D-related vibrational lines, suggesting that $\mathrm{H}$ is released from $V_{\mathrm{Ga}}^{\mathrm{ib}}$ and forms IR-inactive species while $V_{\mathrm{Ga}}^{\mathrm{ib}}$ are still present in the samples. The combination of data from each technique implies that both hydrogen and (split) Ga vacancies, together with their mutual passivation, have a strong influence on the electrical conductivity in $n$-type $\beta-\mathrm{Ga}_{2} \mathrm{O}_{3}$.

\section{METHODS}

The $\beta-\mathrm{Ga}_{2} \mathrm{O}_{3}$ bulk single crystals studied in this work were grown by the edge-defined film-fed growth method (Tamura Corporation). All samples were single side polished and had the (201) surface orientation. $N$-type samples doped with $\mathrm{Sn}$ (carrier concentration of $8 \times 10^{18} \mathrm{~cm}^{-3}$ ) were used for PAS and SIMS measurements. However, the strong free-carrier absorption in these samples prevented IR transmittance measurements of the spectral region where O-D stretching vibrations occur. Therefore, unintentionally-doped (UID) samples (carrier concentration of $1 \times 10^{18} \mathrm{~cm}^{-3}$ ) with either the (201) or (010) surface orientation were used for FT-IR measurements. The different surface orientations were used to ensure all possible O-D dipole moments could be detected.

To study irradiation-generated vacancies, Sn-doped samples were implanted from the polished surface at room temperature with $1.1 \mathrm{MeV} \mathrm{D}^{+}$ions with a fluence of $2 \times 10^{15} \mathrm{~cm}^{-2}$. The UID samples used for FT-IR measurements were implanted with the same fluence but at a lower energy $(200 \mathrm{keV})$. The D implantation peak in the $1.1 \mathrm{MeV}$ $\mathrm{D}^{+}$-implanted samples is located approximately $8 \mu \mathrm{m}$ below the sample surface, leading to a fairly homogeneous damage profile up to $4 \mu \mathrm{m}$. To investigate the effects of annealing, the irradiated samples were annealed in air inside a tube furnace at temperatures between $300^{\circ} \mathrm{C}$ and $625^{\circ} \mathrm{C}$ for 30 minutes.

Doppler broadening of positron-electron annihilation radiation was measured similarly to Ref. 25] with a variable energy slow positron beam. We note here that the resolution of the high-purity Ge detector was $1.25 \mathrm{keV}$ at $511 \mathrm{keV}$, the detector was mounted perpendicular to the beamline and the detectorsample distance is $\sim 3 \mathrm{~cm}$. The $S$ and $W$ parameter integration windows were set as $0-0.45$ a.u. $1.5-4.1$ a.u., respectively. A second $\mathrm{W}$ parameter was defined as $2.0-4.1$ a.u. and re- ferred to as $W 2$ throughout this work, similarly to Ref. 25 , We show data obtained at a positron implantation energy of $25 \mathrm{keV}$, where the effect of surface annihilations was insignificant and the stopping depths are less than $3 \mu \mathrm{m}$, staying within the homogeneous defect profile generated by the $\mathrm{D}$ implantation. $5 \times 10^{6}$ counts were accumulated in the $511 \mathrm{keV}$ annihilation peak in each spectrum and the statistical error estimates of the acquired $S, W$ and $W 2$ parameters are approximately $2 \times 10^{-4}, 1 \times 10^{-4}$ and $5 \times 10^{-5}$, respectively.

To check how the formation of complexes with Sn may affect the positron signals of Ga vacancy defects we performed first-principles calculations fully consistent with earlier work, described in detail in Ref. 24. We considered the experimentally-observed $2 V_{\mathrm{Ga} 1}-\mathrm{Sn}_{\mathrm{ib}}$ and $2 V_{\mathrm{Ga} 1}-\mathrm{Sn}_{\mathrm{ic}}$ complexes in the -2 charge state, and their hydrogenated counterparts $\left(2 V_{\mathrm{Ga} 1}-\mathrm{Sn}_{\mathrm{ib}}-2 \mathrm{H}\right.$ and $2 V_{\mathrm{Ga} 1}-\mathrm{Sn}_{\mathrm{ic}}-2 \mathrm{H}$, respectively) at zero charge state, in monoclinic 160-atom supercells. ${ }^{4 / 20 \mid 24}$ Figure 1 shows the crystallographic orientations along which the experimental and theoretically calculated $(S, W, W 2)$ parameters are determined.
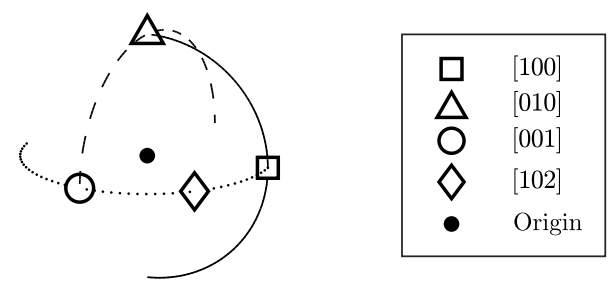

FIG. 1: The notation of the measurement directions for the $(S, W, W 2)$ parameters. The markers represent the given lattice directions and the full, dashed and dotted lines scans along the three "geodesics" which connect [100], [010] and [001] lattice directions. ${ }^{24}$

FT-IR measurements were performed at $5.0 \mathrm{~K}$ at a spectral resolution of $0.5 \mathrm{~cm}^{-1}$ and with the IR beam at normal incidence on the polished sample surface. The samples were cooled in He exchange gas in a Janis PTSHI-950-5 low vibration, pulse tube cryostat equipped with $\mathrm{ZnSe}$ windows. The IR spectra were acquired using a Bruker IFS 125HR spectrometer equipped with a globar source, a $\mathrm{KBr}$ beam splitter, and a liquid nitrogen-cooled InSb detector. The spectra were corrected to remove the sloping baseline arising from absorption due to free carriers and from scattering at the rough back surface. A calibration of the effective charge, $q$, for the 2546$\mathrm{cm}^{-1}-V_{\mathrm{Ga}}^{\mathrm{ib}}-2 \mathrm{D}$ line (at $5 \mathrm{~K}$ ) is presented in Section III. C, using the equation,, 33

$$
q^{2}=\frac{2.303 m n c^{2}}{\pi N d} \int A(\bar{v}) d \bar{v}
$$

where $m$ is the mass of the oscillating impurity (D), $n$ is the refractive index at the wavenumber where the impurity oscillates, $c$ is the speed of light, $N$ is the concentration of $V_{\mathrm{Ga}}^{\mathrm{ib}}-2 \mathrm{D}$, and $d$ is the thickness of the implanted region.

Concentration versus depth profiles of $\mathrm{D}$ were measured using SIMS after post-implantation heat treatments at tempera- 
tures between 300 and $625^{\circ} \mathrm{C}$. The detection limit of D in the SIMS measurements was $<5 \times 10^{15} \mathrm{~cm}^{-3}$, with a vacuum of $<1 \times 10^{-9}$ mTorr in the sample chamber. Further details about the SIMS setup and the measurements can be found in Ref. 32. In general, the electrical conductivity at the surface of the as-implanted samples was too low to permit SIMS measurements. Post-implantation annealing restored the conductivity to a level such that SIMS measurements were possible on the Sn-doped samples, but generally not on the UID samples. However, one SIMS measurement was obtained for a Dimplanted (201) UID sample after subsequent 30-min anneals at $300{ }^{\circ} \mathrm{C}$ and $400{ }^{\circ} \mathrm{C}$. The integrated $\mathrm{D}$ concentration-depth profile from this measurement gives the quantity $N d$ used in equation (1).

\section{RESULTS}

\section{A. Positron annihilation spectroscopy}

Figure 2 shows the $(S, W)$ and $(S, W 2)$ parameters measured in the as-received, irradiated and annealed $\beta-\mathrm{Ga}_{2} \mathrm{O}_{3}$ samples in the [010] and [102] lattice directions. Clearly, the irradiation increases the $S$ and decreases the $W$ and $W 2$ parameters irrespective of measurement direction, indicative of introduced vacancy-type defects. Annealing at $300{ }^{\circ} \mathrm{C}$ effectively doubles the original effect of the irradiation on the $(S, W, W 2)$ parameters. After annealing at $625^{\circ} \mathrm{C}$, the $(S, W, W 2)$ parameters are very close to those of the as-received samples, suggesting recovery of the irradiation-induced vacancy defects.

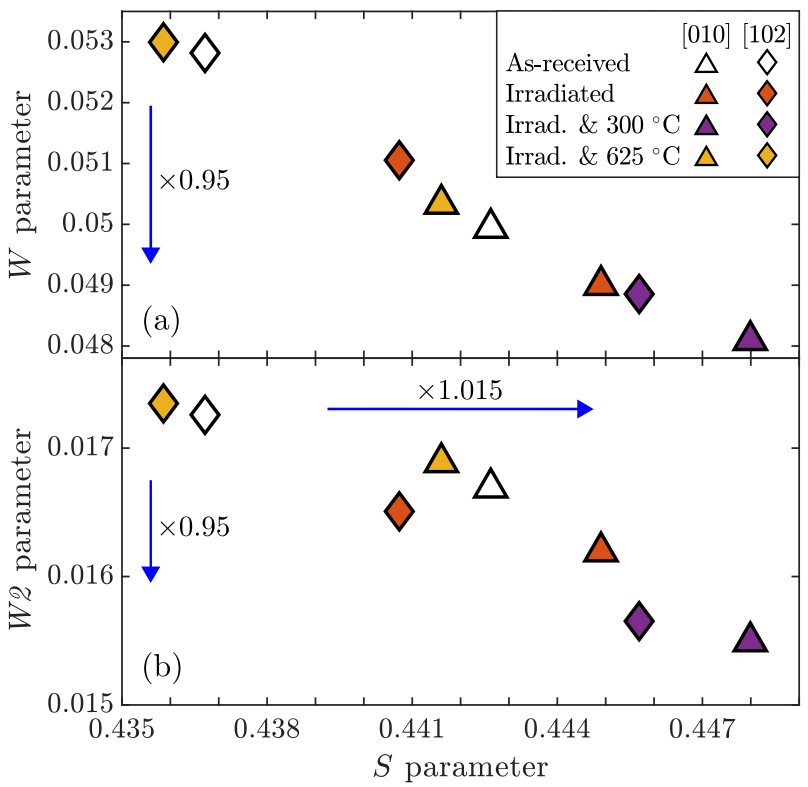

FIG. 2: (a) $(S, W)$ parameters and (b) $(S, W 2)$ parameters measured in the as-received, irradiated and annealed samples in the [010] (triangles) and [102] lattice directions (diamonds). Blue scale bars are drawn to quite the eye. The error estimates are smaller than the markers and not drawn.
Interestingly, the $(S, W, W 2)$ parameters evolve clearly more in the [102] than in the [010] direction after irradiation and annealing, equivalent to a significant change in the degree of anisotropy in the signals. In the as-received and $625^{\circ} \mathrm{C}$ annealed samples, the overall anisotropy in the $S$ parameter is roughly 1.015 , defined as the the highest (lowest) value divided by the lowest (highest) value of the $S(W, W 2)$ parameter, as discussed in Ref. 24. In the $W$ and $W 2$ parameters the anisotropy is 0.95 and 0.96 , respectively. After irradiation, the anisotropy in $S$ and $W$ is reduced by roughly $30 \%$, followed by a reduction of similar magnitude after the $300{ }^{\circ} \mathrm{C}$ anneal. The anisotropy in the $W 2$ parameter is reduced much more, essentially vanishing after the $300^{\circ} \mathrm{C}$ anneal. Analysis of the Doppler broadening lineshapes and the $(S, W, W 2)$ parameters suggests the data of the irradiated sample is a roughly $50 / 50$ superposition of the data of the as-received and $300{ }^{\circ} \mathrm{C}$ annealed samples, meaning that the vacancy signal introduced in the irradiated sample is enhanced after the $300{ }^{\circ} \mathrm{C}$ anneal.

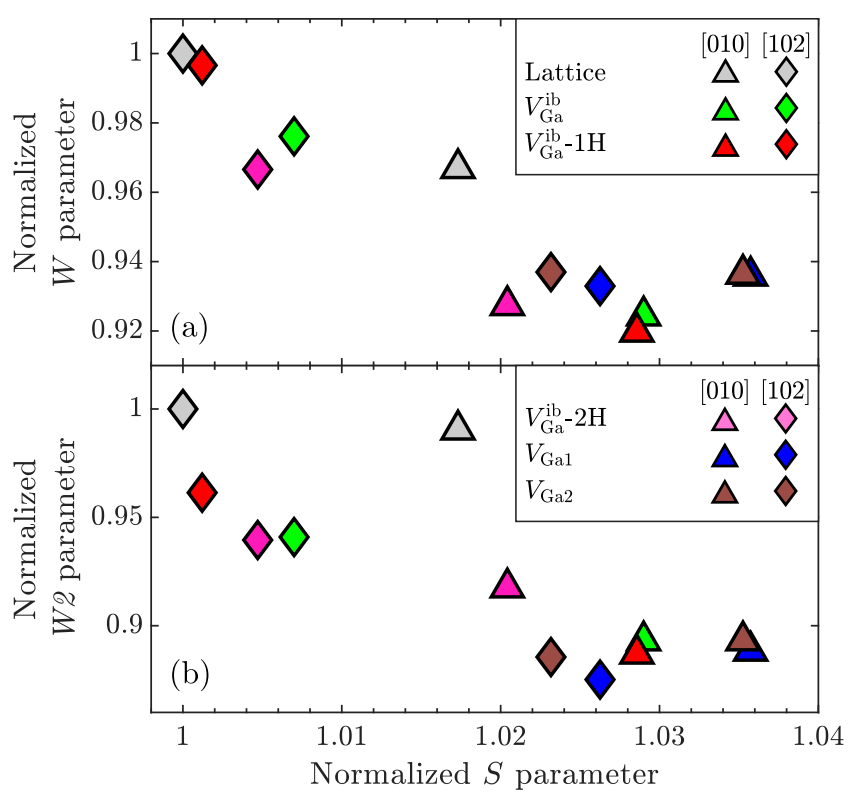

FIG. 3: (a) $(S, W)$ parameters and (b) $(S, W 2)$ parameters calculated for the $V_{\mathrm{Ga}}^{\mathrm{ib}}$ and $V_{\mathrm{Ga}}$ defects in the [010] (triangles) and [102] lattice directions (diamonds). The data in the [102] lattice direction are averaged in the plane spanned by the [100] and [001] lattice directions to take into account the aspect ratio of the positron beam and the effect of the unit cell, described in detail in Ref. 24.

The evolution of the experimental $(S, W, W 2)$ parameters can be compared to the theoretically predicted parameters of various types of defects. Figure 3 shows the calculated $(S, W)$ and $(S, W 2)$ parameters projected in the [010] and [102] lattice directions for $V_{\mathrm{Ga}}^{\mathrm{ib}}$ with 0-2 hydrogen atoms and both unrelaxed $V_{\mathrm{Ga}}$ defects, according to Ref. 24. The differences in the typical behavior of the anisotropy visible in the $(S, W, W 2)$ between the split Ga vacancies and unrelaxed Ga vacancies is notably similar to the experimentally observed difference between as-received (and $625^{\circ} \mathrm{C}$ annealed) and the 
irradiated and $300^{\circ} \mathrm{C}$ samples. This suggests that unrelaxed $\mathrm{Ga}$ vacancies are introduced in the irradiation. Note that the positron signals of the as-received samples are dominated by the split Ga vacancies, as shown in Ref. 25.

To check the possible effect of $\mathrm{Sn}$ replacing $\mathrm{Ga}$ as the center interstitial atom in the split Ga vacancies ${ }^{20}$ on the positron annihilation signals, we calculated the full anisotropy in the $i b$ and ic split Sn-Ga vacancies with and without hydrogen. The results are shown in Fig. 4, and compared to their Sn-free counterparts calculated in Ref. 24. It is clear that the effect of Sn on the $(S, W)$ parameters is minimal, slightly increasing the overall anisotropy, but not changing its nature in any way. Hence the evolution of the experimental positron annihilation signals in irradiation and annealing should not be related to interactions between $\mathrm{Sn}$ and Ga vacancies. Note that the effect of $\mathrm{Sn}$ on the calculated positron lifetimes (not shown) is also minimal, resulting in at most 2 ps increase compared to the values in Ref. 24 .

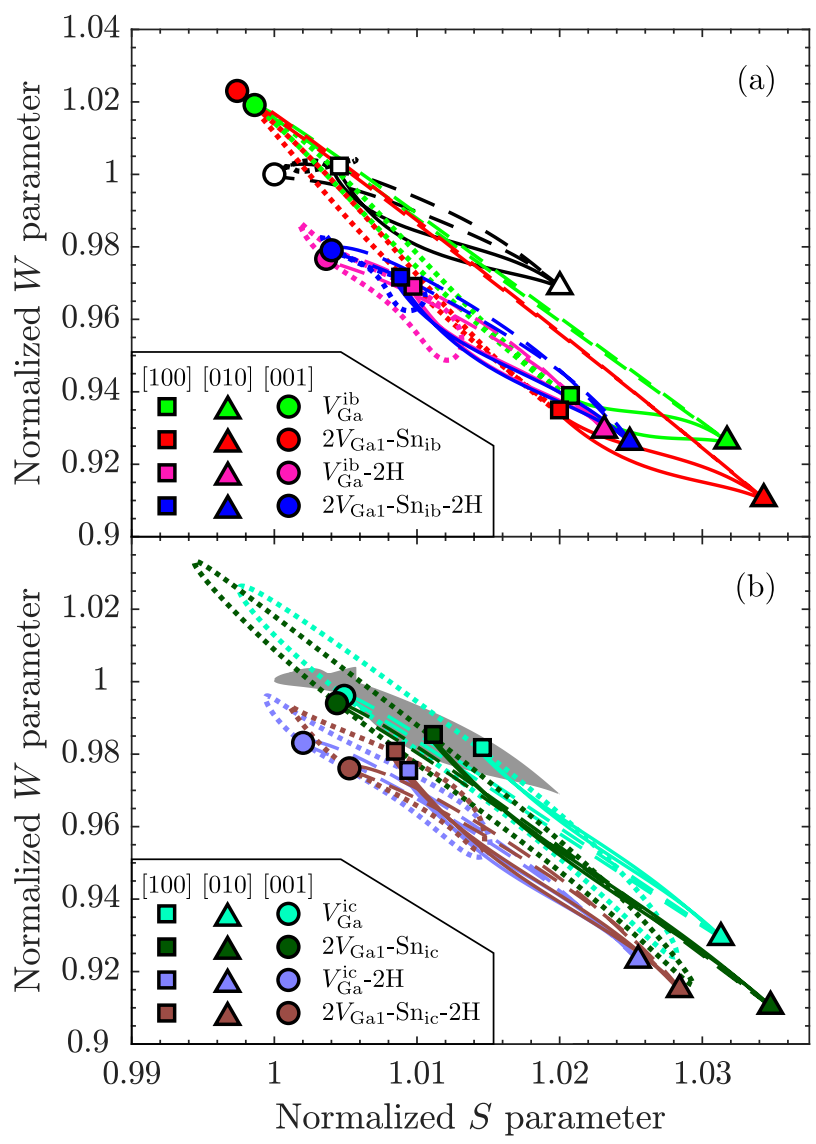

FIG. 4: Calculated $(S, W)$ parameters of $V_{\mathrm{Ga}}^{\mathrm{ib}}, V_{\mathrm{Ga}}^{\mathrm{ib}}-2 \mathrm{H}, V_{\mathrm{Ga}}^{\mathrm{ic}}$ and $V_{\mathrm{Ga}}^{\mathrm{ic}}-2 \mathrm{H}$ with and without $\mathrm{Sn}$. The $\beta-\mathrm{Ga}_{2} \mathrm{O}_{3}$ lattice signal is shown for comparison ${ }^{24}$ in (a) with white symbols and in (b) as a grey shadow. The full, dashed and dotted lines follow the notation introduced in Fig. 1. Complexing with Sn has a negligible effect on the $(S, W)$ parameters of split $\mathrm{Ga}$ vacancies.

\section{B. FT-IR spectroscopy}

Figure 5 shows IR absorbance spectra for a (201)-oriented $\beta-\mathrm{Ga}_{2} \mathrm{O}_{3}$ sample in the spectral region where O-D stretching vibrations occur after $\mathrm{D}$ implantation and subsequent heat treatments. No O-D or O-H-related vibrational lines were detected in the as-received samples. The as-implanted sample exhibits several absorption lines that are positioned at 2518, $2546,2567,2578,2591$, and $2632 \mathrm{~cm}^{-1}$. The dominant line at $2546 \mathrm{~cm}^{-1}$ originates from $V_{\mathrm{Ga}}^{\mathrm{ib}}-2 \mathrm{D}{ }^{26 / 27}$ The $300^{\circ} \mathrm{C}$ annealing increases the intensity of the $2546 \mathrm{~cm}^{-1}$ line at the expense of the intensities of the other O-D lines. Subsequent anneals at higher temperatures up to $700{ }^{\circ} \mathrm{C}$ cause the intensity of the $2546 \mathrm{~cm}^{-1}$ line to decrease considerably, and also result in the appearance of absorption lines at 2476, 2532, 2542, and $2639 \mathrm{~cm}^{-1}$. The assignment of these new absorption lines to O-D stretching modes was recently reported by Portoff et $a l{ }^{29}$ Importantly, the spectra in Figure 5 show how the dominant O-D species in the implanted region is controlled by the post-implantation annealing temperature. Similar results are obtained for the (010)-oriented sample, i.e., none of the vibrational lines have a dipole moment oriented along [010].

The integrated absorbance of the 2546- $\mathrm{cm}^{-1} V_{\mathrm{Ga}}^{\mathrm{ib}}-2 \mathrm{D}$ line for probing light along the [102] axis after the $300^{\circ} \mathrm{C}$ anneal is $0.023 \mathrm{~cm}^{-1}$. The dipole moment of this defect is at an angle of $15^{\circ}$ from the [102] axis, which results in a correction to the integrated absorbance, to $0.025 \mathrm{~cm}^{-1}$. The D concentration-depth profile (not shown) for this sample yields $N_{D} d=4.07 \times 10^{15} \mathrm{~cm}^{-2}$. This value should be divided by two because the defect in question contains two $\mathrm{D}$ atoms. Assuming an isotropic medium for the refractive in$\operatorname{dex}(n=1.88)$ and that all of the $\mathrm{D}$ is trapped at $V_{\mathrm{Ga}}^{\mathrm{ib}}$ after the $300{ }^{\circ} \mathrm{C}$ anneal, the effective charge (for probing light along [102] $)$ is determined to be $(0.47 \pm 0.05)$ e. The corresponding concentration of $V_{\mathrm{Ga}}^{\mathrm{ib}}-2 \mathrm{D}$ (in the implantation peak) after the $300{ }^{\circ} \mathrm{C}$ anneal is $1.3 \times 10^{19} \mathrm{~cm}^{-3}$, which is in excellent agreement with the value estimated from the implantation conditions (fluence of $2 \times 10^{15} \mathrm{~cm}^{-2}$ and an implanted region with thickness of $1.5 \mu \mathrm{m})$. The detection limit is estimated to be $\sim 1 \times 10^{17} \mathrm{~cm}^{-3}$ in a $1.5 \mu \mathrm{m}$ thick layer (the detection limit scales inversely proportional to the layer thickness).

\section{Secondary ion mass spectrometry}

Figure 6 shows D concentration versus depth profiles in D-implanted, Sn-doped $\beta-\mathrm{Ga}_{2} \mathrm{O}_{3}$ samples after postimplantation heat treatments at 300,525 , and $600^{\circ} \mathrm{C}$. The $300{ }^{\circ} \mathrm{C}$ anneal shows a well-defined implantation peak located approximately $8 \mu \mathrm{m}$ below the surface with a maximum D concentration of $4 \times 10^{19} \mathrm{~cm}^{-3}$. Diffusion of $\mathrm{D}$ into the bulk $\beta-\mathrm{Ga}_{2} \mathrm{O}_{3}$ is observed after the $525^{\circ} \mathrm{C}$ anneal, whereas noticeable diffusion towards the surface is also seen after the $600{ }^{\circ} \mathrm{C}$ anneal. Reinertsen et al. ${ }^{32}$ showed recently that the D concentration-depth profiles can be modeled by trap-limited diffusion, where the diffusion of $\mathrm{D}$ is controlled by its capture and release from traps present either in the as-received samples or after ion implantation. The height of the diffusion 


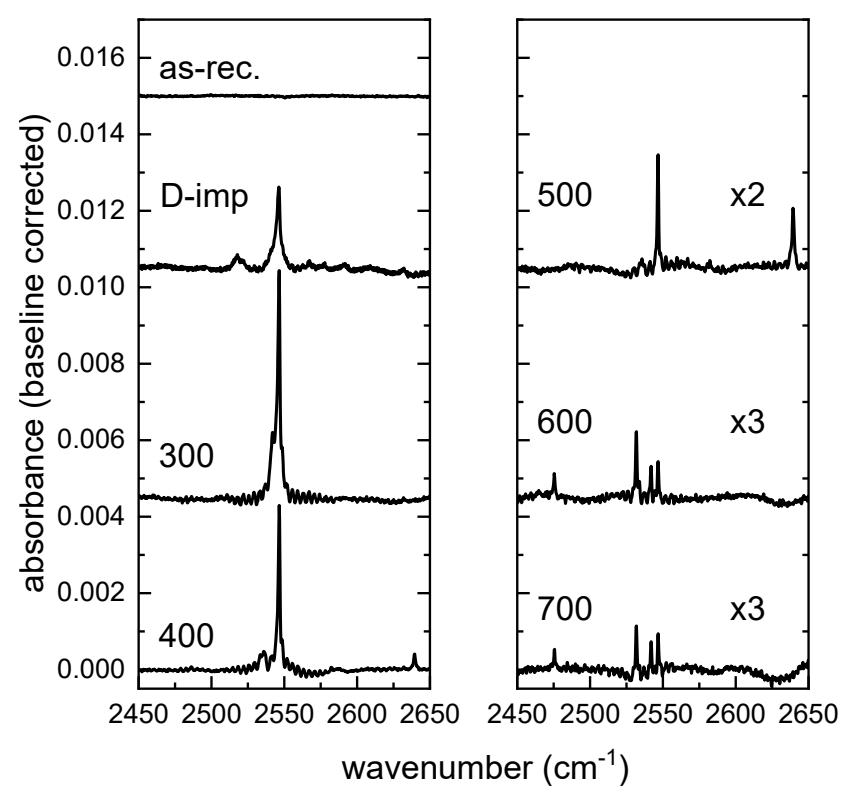

FIG. 5: Baseline-corrected IR absorbance spectra at $5 \mathrm{~K}$ of a (201)-oriented $\beta-\mathrm{Ga}_{2} \mathrm{O}_{3}$ sample in the as-received state and after implantation and subsequent isochronal anneals (30 min) at temperatures between 300 and $700{ }^{\circ} \mathrm{C}$.

front on either side of the implantation peak can be used to estimate the concentration of trap(s) present in the sample. A trap concentration (between the surface and the implantation peak) in the order of $10^{18} \mathrm{~cm}^{-3}$ is estimated from the $600^{\circ} \mathrm{C}$ profile shown in Figure 6 Notably, the SIMS profiles imply that the positron measurements do not probe the region with implanted D even after the highest-temperature annealing.

\section{DISCUSSION}

The positron results show that as-received samples show the strongest Doppler broadening signal anisotropy and, upon irradiation, a larger change in $(S, W)$ parameters in [102] than in [010] lattice direction, which are both in agreement with the theoretica ${ }^{24}$ and experimental ${ }^{25]}$ positron work where a high concentration of split Ga vacancies of type $V_{\mathrm{Ga}}^{\mathrm{ib}}$ or $V_{\mathrm{Ga}}^{\text {ic }}$ were identified in as-received $\beta-\mathrm{Ga}_{2} \mathrm{O}_{3}$ single crystals. The $\mathrm{D}$ implantation introduces vacancy defects with a higher $S$ parameter (lower $W$ and $W 2$ ) and a significantly smaller Doppler signal anisotropy than those in the as-received sample, in agreement with the annihilation parameters predicted for $V_{\mathrm{Ga}}$ and $V_{\mathrm{Ga} 2}$. However, first-principles calculations ${ }^{10 / 34}$ predict that unrelaxed $V_{\mathrm{Ga}}$, i.e., $V_{\mathrm{Ga} 1}$ and $V_{\mathrm{Ga} 2}$, are metastable configurations, and should already be able to overcome the relatively low barriers $(<0.5 \mathrm{eV})$ to relax into the split configurations at room temperature. Furthermore, the calculated O-D stretch modes of D-containing unshifted $V_{\mathrm{Ga} 1}$ and $V_{\mathrm{Ga} 2}$ defects should be polarized along the [010] lattice vector ${ }^{262735}$, but the O$\mathrm{D}$ vibrational lines observed in implanted samples (ours and

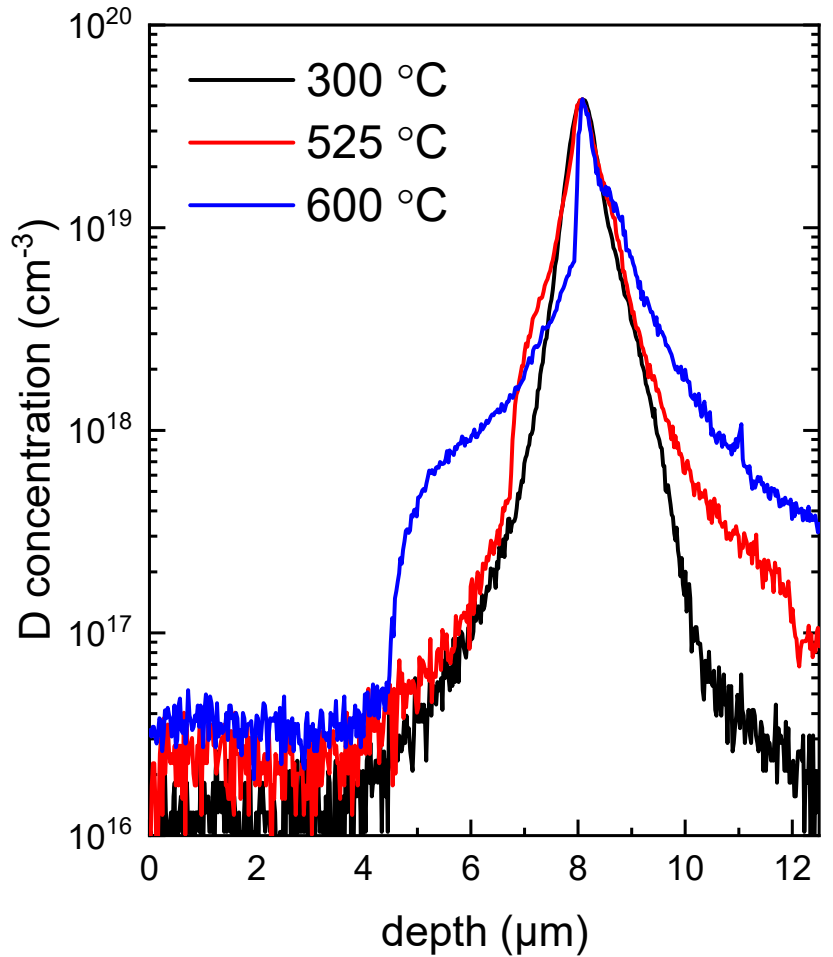

FIG. 6: D concentration-depth profiles for a (201)-oriented $\beta-\mathrm{Ga}_{2} \mathrm{O}_{3}$ single crystal after $\mathrm{D}$ implantation and subsequent isochronal anneals (30 $\mathrm{min}$ ) at 300,525 , and $600^{\circ} \mathrm{C}$.

Ref. 29) are not polarized along [010]. Recent first-principles calculations by Fowler et al. showed that $\mathrm{H}$ trapping and relaxation processes in $\beta-\mathrm{Ga}_{2} \mathrm{O}_{3}$ make it highly unlikely that any of the hydrogenated unshifted $V_{\mathrm{Ga}}$ will occur. ${ }^{355}$ Interestingly, recent experimental ${ }^{16}$ and theoretical work ${ }^{19}$ have suggested that $V_{\mathrm{Ga}}-V_{\mathrm{O}}$ divacancy complexes could give rise to the so-called E2* level observed using DLTS in $\beta-\mathrm{Ga}_{2} \mathrm{O}_{3}$ single crystals after $\mathrm{H}$ - and He- irradiation. Importantly, the positron annihilation signature of a cation vacancy is expected to remain relatively unchanged compared to its complex with a small anion vacancy 25136. Therefore, the changes to the positron signals after irradiation could originate from creation of $V_{\mathrm{Ga}}-V_{\mathrm{O}}$ complexes containing $V_{\mathrm{Ga} 1}$ and $V_{\mathrm{Ga} 2}$.

Samples annealed at $300{ }^{\circ} \mathrm{C}$ can be probed by SIMS, PAS, and FT-IR. The SIMS profile (black curve) shown in Figure 6 shows a well-defined D implantation peak centered approximately $8 \mu \mathrm{m}$ below the sample surface, which is consistent with SRIM simulations 37 . For positrons, the $300{ }^{\circ} \mathrm{C}$ annealing further increases $S$ parameter (decreases $W$ and $W 2$ ) and reduces the Doppler signal anisotropy. A thorough analysis of the Doppler spectral shape suggests that this change is due increased positron trapping at the irradiation-generated $V_{\mathrm{Ga} 1}$ and $V_{\mathrm{Ga} 2}$-like defects, rather than a change in their structure or creation of new $V_{\mathrm{Ga}}$ defects. The observed anisotropy in the $300^{\circ} \mathrm{C}$-annealed sample is also comparable to the anisotropy predicted for $V_{\mathrm{Ga}}$ - and $V_{\mathrm{Ga} 2}$-like defects, which suggests that the positron trapping to these vacancies could be near satu- 
ration $\left(>1 \times 10^{18} \mathrm{~cm}^{-3}\right)$. One plausible explanation for the increased trapping at the irradiation-induced $V_{\mathrm{Ga}}{ }^{-}$and $V_{\mathrm{Ga} 2}-$ like defects is that other positron traps, e.g., split $V_{\mathrm{Ga}}$, become less efficient due to $\mathrm{H}$ passivation. Indeed, theoretical work ${ }^{11 / 38}$ suggests that twofold H/D passivation reduces the positron trapping coefficient of cation vacancies $\left(V_{\mathrm{Ga}}^{\mathrm{ib}}-2 \mathrm{H}\right)$ by a factor of $\sim 10$ due to the reduced remaining open volume and the more positive charge. Therefore, the higher fraction of positrons that are trapped at $V_{\mathrm{Ga} 1}$ - or $V_{\mathrm{Ga} 2}$-like defects enhances their contribution to the positron signal. Ingebritsen et al ${ }^{4}$ proposed passivation of $V_{\mathrm{Ga}}$ acceptors by $\mathrm{H}$ as a potential explanation for the charge carrier recovery observed in $\mathrm{H}^{+}$-implanted and annealed $\beta-\mathrm{Ga}_{2} \mathrm{O}_{3}$ single crystals. Indeed, passivation of split $V_{\mathrm{Ga}}$ by $\mathrm{D}$ is qualitatively consistent with the FT-IR data in Figure 5, which shows an increase in the intensity of the $2546.4-\mathrm{cm}^{-1} V_{\mathrm{Ga}}^{\mathrm{ib}}-2 \mathrm{D}$ line by almost a factor of 3. This increase is accompanied by the disappearance of other O-D vibrational lines seen after implantation. Evidently, the charge carrier recovery does not require the removal of unrelaxed $V_{\mathrm{Ga}}$ defects, but rather the passivation of $V_{\mathrm{Ga}}^{\mathrm{i}}$-type defects, and suggests that $\mathrm{H}$ plays a significant role in determining the degree of compensation, as proposed in Refs. 12, 13, and 24

The above-mentioned interpretation of the positron data after the $300{ }^{\circ} \mathrm{C}$ anneal requires a concentration of $\mathrm{H}$ or $\mathrm{D}$ in the order of $\sim 10^{18} \mathrm{~cm}^{-3}$ in the region probed by positrons. The SIMS data in Figure 6 show that the concentration of $\mathrm{D}$ in the damage track is nearly two orders of magnitude lower than this, which implies that the $\sim 10^{18} \mathrm{~cm}^{-3}$ is determined by $\mathrm{H}$ present in the as-grown material. Hydrogen and silicon were proposed to be the two main impurities responsible for the unintentional n-type conductivity in $\beta-\mathrm{Ga}_{2} \mathrm{O}_{3}$, 239]44] Indeed, Czochralski-grown $\beta-\mathrm{Ga}_{2} \mathrm{O}_{3}$ single crystals were shown to contain substantial contributions to the free electron concentration arising from $\mathrm{H}$ incorporated during growth and at concentrations $\left(10^{17}-10^{18} \mathrm{~cm}^{-3}\right)$ that are consistent with the PAS results ${ }^{40}$. As stated above, the strong free-carrier absorption prevents investigation of the $3437 \mathrm{~cm}^{-1} V_{\mathrm{Ga}}^{\mathrm{ib}}-2 \mathrm{H}$ line in the Sn-doped samples, but it is completely absent in the UID samples even after post-implantation annealings. If one assumes that the residual $\mathrm{H}$ concentration is similar in both Sn-doped and UID samples, then the FT-IR data suggest a residual $\mathrm{H}$ concentration well below that needed to explain the PAS results. However, we cannot exclude the possibility that there is a difference in the residual $\mathrm{H}$ concentration between the UID and Sn-doped samples, or that $\mathrm{H}$ is present as an IRinactive species, e.g., $\mathrm{H}_{2}$. Further investigations are needed to clarify this discrepancy.

The positron data after the $625^{\circ} \mathrm{C}$ anneal are similar to the data for the as-received samples. The disappearance of the irradiation-generated $V_{\mathrm{Ga}}$ signals implies that these defects are either partially or entirely removed by the anneal, which could occur either by diffusing out of the probing region or by relaxing to the energetically-favorable split $V_{\mathrm{Ga}}$ configurations 3 (110. We analyze the thermal annealing kinetics similarly as Kyrtsos et al ${ }^{34}$ by assuming a typical phonon frequency of $1 \times 10^{13} \mathrm{~Hz}$ as an approximation for the attempt frequency and $1 \mathrm{~Hz}$ as the estimate reaction rate where recov- ery is visible. We observe the recovery of regular vacancies at a temperature between $300^{\circ} \mathrm{C}$ and $625^{\circ} \mathrm{C}$. These temperatures correspond to $1.5 \mathrm{eV}$ and $2.3 \mathrm{eV}$, respectively, hence the migration barrier for the irradiation-generated vacancy defects can be estimated as $(1.9 \pm 0.4) \mathrm{eV}$. The $2546 \mathrm{~cm}^{-1}$ line disappears from the spectrum, and weak O-D lines appear in its vicinity, which have been speculated to arise from complexes between $V_{\mathrm{Ga}}^{\mathrm{ib}}-2 \mathrm{D}$ and other defects created by implantation ${ }^{29}$. The SIMS results in Figure 6 show that the majority of the implanted D is still present in the sample, and, therefore, the outdiffusion of D cannot explain the disappearance of the 2546 $\mathrm{cm}^{-1}$ line. The positron results indicate that defects with signatures matching those of split $V_{\mathrm{Ga}}$ are still present after the $625^{\circ} \mathrm{C}$ annealing, which is in agreement with the suggestion by Ref. 29. Therefore, the combination of positron annihilation, SIMS, and FT-IR data suggest that D (or whole $V_{\mathrm{Ga}}^{\mathrm{ib}}-2 \mathrm{D}$ defects) are present in a different, IR-inactive configuration after the $625^{\circ} \mathrm{C}$ anneal. These configurations are either not able to trap positrons efficiently, and therefore do not affect the positron signal, or have positron signals that closely resemble those of split $V_{\mathrm{Ga}}$.

Lastly, we note that the observed evolution of split and unrelaxed $\mathrm{Ga}$ vacancies and their interactions with hydrogen provide strong evidence for these point defects determining the degree of compensation in $n$-type $\beta-\mathrm{Ga}_{2} \mathrm{O}_{3}$. Importantly, as observed already earlier, the originally $n$-type conductive $\beta-\mathrm{Ga}_{2} \mathrm{O}_{3}$ samples become highly resistive or even semi-insulating after the irradiation, which renders electrical measurements (e.g., DLTS) impossible ${ }^{4}$ and causes charging problems in SIMS. Annealing at $300^{\circ} \mathrm{C}$ restores the conductivity, but as seen in our present results, the irradiationinduced $\mathrm{Ga}$ vacancies have not recovered. This means that the compensation caused by the introduction of unrelaxed $\mathrm{Ga}$ vacancies is canceled out by the motion of hydrogen into vacancy defects. Further investigations are required to identify the detailed mechanisms behind the compensation, but it is clear that the interplay of (split) Ga vacancies and hydrogen plays a decisive role.

\section{SUMMARY}

In summary, positron annihilation, FT-IR, and SIMS have been combined to study the evolution of $V_{\mathrm{Ga}}$ and hydrogen in $\beta-\mathrm{Ga}_{2} \mathrm{O}_{3}$ single crystals after D-implantation and subsequent thermal annealing. The positron annihilation data suggest that the D-implantation creates defects that contain unrelaxed $V_{\mathrm{Ga}}$, and experimental and theoretical results in the literature point towards $V_{\mathrm{Ga}}-V_{\mathrm{O}}$ divacancies as the possible candidates for these species. The presence of several types of $V_{\mathrm{Ga}}$-related defects is also confirmed by FT-IR from the presence of multiple O-D-related vibrational lines present in the as-implanted sample. The $300{ }^{\circ} \mathrm{C}$ anneal enhances the intensity of the $2546-\mathrm{cm}^{-1} V_{\mathrm{Ga}}^{\mathrm{ib}}-2 \mathrm{D}$ vibrational line by a factor of 3 , which suggests that the other O-D-related defects provide a source of D that can passivate $V_{\mathrm{Ga}}^{\mathrm{ib}}$ created during implantation or present in the as-received sample. The passivation of $V_{\mathrm{Ga}}^{\mathrm{ib}}$ by $\mathrm{D}$ suppresses its ability to capture positrons, thereby allow- 
ing their capture by $V_{\mathrm{Ga} 1}$ - and $V_{\mathrm{Ga} 2}$-related defects. Although these techniques are in qualitative agreement for this explanation, additional investigations are needed to clarify the apparent quantitative discrepancy. The anneal at $625^{\circ} \mathrm{C}$ returns the positron signals back to the levels in the as-received samples, and also results in relatively small but visible diffusion of D. The lack of D out-diffusion, the still remaining strong split Ga vacancy -associated positron signal, the almost complete removal of the $2546-\mathrm{cm}^{-1}$ line, and the appearance of several new O-D-related vibrational lines are consistent with the release of D from $V_{\mathrm{Ga}}^{\mathrm{ib}}$ to form different, IR-inactive species; the formation of larger defect complexes between $V_{\mathrm{Ga}}^{\mathrm{ib}}-\mathrm{D}$ and other implantation-induced point defects; or a combination of the two. This study provides new insights and considerations for future studies of $V_{\mathrm{Ga}}$-type defects and their interaction with hydrogen. In particular their interplay in determining the degree of compensation of $n$-type $\beta-\mathrm{Ga}_{2} \mathrm{O}_{3}$ appears key to understanding the electrical characteristics of this material.

\section{ACKNOWLEDGMENTS}

We wish to thank Y. K. Frodason for sharing unpublished results about $V_{\mathrm{Ga}}-V_{\mathrm{O}}$ defects. We are also grateful to $\mathrm{M}$. Stavola and W. B. Fowler for sharing their manuscript on H/D implantations in $\beta-\mathrm{Ga}_{2} \mathrm{O}_{3}$ prior to publication, as well as for helpful discussions regarding the calibration of the $V_{\mathrm{Ga}}^{\mathrm{ib}}-2 \mathrm{D}$ vibrational line. The authors gratefully acknowledge the computational resources provided by the CSC (Finnish IT Centre for Science). This work was partially supported by the Academy of Finland Grants No. 285809, No. 315082, and No. 319178. A.K. wishes to thank the Magnus Ehrnrooth foundation for financial support. The work at the Univ. of Oslo was supported by the Research Council of Norway by the Norwegian Micro- and Nano-Fabrication Facility (NorFab No. 295864), the Functionalizing Defects in Advanced Semiconductors (FUNDAMENT No. 251131), and the Research Center for Sustainable Solar Cell Technology (FME SUSOLTECH No. 257639).

\section{AIP PUBLISHING DATA SHARING POLICY}

The data that support the findings of this study are available from the corresponding author upon reasonable request.

\section{REFERENCES}

${ }^{1}$ S. Pearton, J. Yang, P. H. Cary IV, F. Ren, J. Kim, M. J. Tadjer, and M. A. Mastro, "A review of $\mathrm{Ga}_{2} \mathrm{O}_{3}$ materials, processing, and devices," Applied Physics Reviews 5, 011301 (2018).

${ }^{2}$ J. Zhang, J. Shi, D.-C. Qi, L. Chen, and K. H. L. Zhang, "Recent progress on the electronic structure, defect, and doping properties of $\mathrm{Ga}_{2} \mathrm{O}_{3}$," APL Materials 8, 020906 (2020) https://doi.org/10.1063/1.5142999

${ }^{3}$ M. Higashiwaki, H. Murakami, Y. Kumagai, and A. Kuramata, "Current status of $\mathrm{Ga}_{2} \mathrm{O}_{3}$ power devices," Japanese Journal of Applied Physics 55, 1202A1 (2016).
${ }^{4}$ M. E. Ingebrigtsen, A. Y. Kuznetsov, B. G. Svensson, G. Alfieri, A. Mihaila, U. Badstübner, A. Perron, L. Vines, and J. B. Varley, "Impact of proton irradiation on conductivity and deep level defects in $\beta-\mathrm{Ga}_{2} \mathrm{O}_{3}$," APL Materials 7, 022510 (2019) https://doi.org/10.1063/1.5054826

${ }^{5}$ Z. Zhang, E. Farzana, A. R. Arehart, and S. A. Ringel, "Deep level defects throughout the bandgap of (010) $\beta-\mathrm{Ga}_{2} \mathrm{O}_{3}$ detected by optically and thermally stimulated defect spectroscopy," Applied Physics Letters 108, 052105 (2016) https://doi.org/10.1063/1.4941429

${ }^{6}$ E. Farzana, E. Ahmadi, J. S. Speck, A. R. Arehart, and S. A. Ringel, "Deep level defects in $\mathrm{Ge}$-doped (010) $\beta$ - $\mathrm{Ga}_{2} \mathrm{O}_{3}$ layers grown by plasma-assisted molecular beam epitaxy," Journal of Applied Physics 123, 161410 (2018) https://doi.org/10.1063/1.5010608

${ }^{7}$ A. Y. Polyakov, N. B. Smirnov, I. V. Shchemerov, D. Gogova, S. A. Tarelkin, and S. J. Pearton, "Compensation and persistent photocapacitance in homoepitaxial Sn-doped $\beta-\mathrm{Ga}_{2} \mathrm{O}_{3}$," Journal of Applied Physics 123, 115702 (2018) https://doi.org/10.1063/1.5025916

${ }^{\gamma}$ E. Farzana, M. F. Chaiken, T. E. Blue, A. R. Arehart, and S. A. Ringel, "Impact of deep level defects induced by high energy neutron radiation in $\beta-\mathrm{Ga}_{2} \mathrm{O}_{3}$," APL Materials 7, 022502 (2019) https://doi.org/10.1063/1.5054606

${ }^{9}$ C. Zimmermann, V. Rønning, Y. Kalmann Frodason, V. Bobal, L. Vines, and J. B. Varley, "Primary intrinsic defects and their charge transition levels in $\beta-\mathrm{Ga}_{2} \mathrm{O}_{3}$, , Phys. Rev. Materials 4, 074605 (2020)

${ }^{10}$ J. B. Varley, H. Peelaers, A. Janotti, and C. G. V. de Walle, "Hydrogenated cation vacancies in semiconducting oxides,"Journal of Physics: Condensed Matter 23, 334212 (2011)

${ }^{11} \mathrm{~F}$. Tuomisto and I. Makkonen, "Defect identification in semiconductors with positron annihilation: Experiment and theory," Rev. Mod. Phys. 85, 1583-1631 (2013)

${ }^{12}$ E. Korhonen, F. Tuomisto, D. Gogova, G. Wagner, M. Baldini, Z. Galazka, R. Schewski, and M. Albrecht, "Electrical compensation by Ga vacancies in $\mathrm{Ga}_{2} \mathrm{O}_{3}$ thin films," Applied Physics Letters 106, 242103 (2015).

${ }^{13}$ F. Tuomisto, A. Karjalainen, V. Prozheeva, I. Makkonen, G. Wagner, and M. Baldini, "Ga vacancies and electrical compensation in $\beta-\mathrm{Ga}_{2} \mathrm{O}_{3}$ thin films studied with positron annihilation spectroscopy," in Oxide-based Materials and Devices X, Vol. 10919 (International Society for Optics and Photonics, 2019) p. 1091910.

${ }^{14}$ A. Y. Polyakov, N. B. Smirnov, I. V. Shchemerov, E. B. Yakimov, J. Yang, F. Ren, G. Yang, J. Kim, A. Kuramata, and S. J. Pearton, "Point defect induced degradation of electrical properties of $\mathrm{Ga}_{2} \mathrm{O}_{3}$ by $10 \mathrm{MeV}$ proton damage," Applied Physics Letters 112, 032107 (2018) https://doi.org/10.1063/1.5012993

${ }^{15}$ A. Y. Polyakov, N. B. Smirnov, I. V. Shchemerov, E. B. Yakimov, S. J. Pearton, C. Fares, J. Yang, F. Ren, J. Kim, P. B. Lagov, V. S. Stolbunov, and A. Kochkova, "Defects responsible for charge carrier removal and correlation with deep level introduction in irradiated $\beta-\mathrm{Ga}_{2} \mathrm{O}_{3}$," Applied Physics Letters 113, 092102 (2018) https://doi.org/10.1063/1.5049130

${ }^{10}$ C. Zimmermann, E. F. Verhoeven, Y. K. Frodason, P. M. Weiser, J. B. Varley, and L. Vines, "Formation and control of the E2* center in implanted $\beta-\mathrm{Ga}_{2} \mathrm{O}_{3}$ by reverse-bias and zero-bias annealing," Journal of Physics D: Applied Physics 53, 464001 (2020)

${ }^{1 /}$ J. F. Mcglone, Z. Xia, Y. Zhang, C. Joishi, S. Lodha, S. Rajan, S. A. Ringel, and A. R. Arehart, "Trapping effects in $\mathrm{Si} \delta$-doped $\beta$ - $\mathrm{Ga}_{2} \mathrm{O}_{3}$ MESFETs on an Fe-doped $\beta-\mathrm{Ga}_{2} \mathrm{O}_{3}$ substrate," IEEE Electron Device Letters 39, 1042$1045(2018)$

${ }^{18}$ J. F. McGlone, Z. Xia, C. Joishi, S. Lodha, S. Rajan, S. Ringel, and A. R. Arehart, "Identification of critical buffer traps in $\mathrm{Si} \delta$ doped $\beta-\mathrm{Ga}_{2} \mathrm{O}_{3}$ MESFETs," Applied Physics Letters 115, 153501 (2019). https://doi.org/10.1063/1.5118250

${ }^{19}$ Y. K. Frodason, C. Zimmermann, E. F. Verhoeven, P. M. Weiser, L. Vines, and J. B. Varley, "Multistability of isolated and hydrogenated Ga-O divacancies in $\beta$ - $\mathrm{Ga}_{2} \mathrm{O}_{3}$," Phys. Rev. Materials 5, 025402 (2021)

${ }^{20}$ J. M. Johnson, Z. Chen, J. B. Varley, C. M. Jackson, E. Farzana, Z. Zhang, A. R. Arehart, H.-L. Huang, A. Genc, S. A. Ringel, C. G. Van de Walle, D. A. Muller, and J. Hwang, "Unusual formation of point-defect complexes in the ultrawide-band-gap semiconductor $\beta-\mathrm{Ga}_{2} \mathrm{O}_{3}$," Phys. Rev. X 9, 041027 (2019).

${ }^{21}$ H. J. von Bardeleben, S. Zhou, U. Gerstmann, D. Skachkov, W. R. L. Lambrecht, Q. D. Ho, and P. Deák, "Proton irradiation induced defects in $\beta$ $\mathrm{Ga}_{2} \mathrm{O}_{3}$ : A combined EPR and theory study," APL Materials 7, 022521 
(2019) https://doi.org/10.1063/1.5053158

${ }^{22}$ D. Skachkov, W. R. L. Lambrecht, H. J. von Bardeleben, U. Gerstmann, Q. D. Ho, and P. Deák, "Computational identification of Ga-vacancy related electron paramagnetic resonance centers in $\beta-\mathrm{Ga}_{2} \mathrm{O}_{3}$," Journal of Applied Physics 125, 185701 (2019) https://doi.org/10.1063/1.5092626

${ }^{25}$ N. T. Son, Q. D. Ho, K. Goto, H. Abe, T. Ohshima, B. Monemar, Y. Kumagai, T. Frauenheim, and P. Deák, "Electron paramagnetic resonance and theoretical study of gallium vacancy in $\beta-\mathrm{Ga}_{2} \mathrm{O}_{3}$," Applied Physics Letters

117, 032101 (2020) https://doi.org/10.1063/5.0012579

${ }^{24}$ A. Karjalainen, V. Prozheeva, K. Simula, I. Makkonen, V. Callewaert, J. B. Varley, and F. Tuomisto, "Split Ga vacancies and the unusually strong anisotropy of positron annihilation spectra in $\beta-\mathrm{Ga}_{2} \mathrm{O}_{3}$," Phys. Rev. B 102, 195207 (2020)

${ }^{25}$ A. Karjalainen, I. Makkonen, J. Etula, K. Goto, H. Murakami, Y. Kumagai, and F. Tuomisto, "Split Ga vacancies in $n$-type and semi-insulating $\beta-\mathrm{Ga}_{2} \mathrm{O}_{3}$ single crystals," Applied Physics Letters 118, 072104 (2021) https://doi.org/10.1063/5.0033930

${ }^{26}$ P. Weiser, M. Stavola, W. B. Fowler, Y. Qin, and S. Pearton, "Structure and vibrational properties of the dominant $\mathrm{OH}$ center in $\beta-\mathrm{Ga}_{2} \mathrm{O}_{3}$," Appl. Phys. Lett. 112, 232104 (2018).

${ }^{27}$ Y. Qin, M. Stavola, W. B. Fowler, P. Weiser, and S. J. Pearton, "Hydrogen centers in $\beta-\mathrm{Ga}_{2} \mathrm{O}_{3}$ : Infrared spectroscopy and density functional theory,' ECS Journal of Solid State Science and Technology 8, Q3103-Q3110 (2019)

${ }^{28}$ J. R. Ritter, J. Huso, P. T. Dickens, J. B. Varley, K. G. Lynn, and M. D. McCluskey, "Compensation and hydrogen passivation of magnesium acceptors in $\beta-\mathrm{Ga}_{2} \mathrm{O}_{3}$," Applied Physics Letters 113, 052101 (2018) https://doi.org/10.1063/1.5044627

${ }^{29}$ A. Portoff, A. Venzie, Y. Qin, M. Stavola, W. B. Fowler, and S. J. Pearton, "Vibrational properties of oxygen-hydrogen centers in $\mathrm{H}^{+}$- and $\mathrm{D}^{+}$-implanted $\mathrm{Ga}_{2} \mathrm{O}_{3}$," ECS Journal of Solid State Science and Technology 9, 125006 (2020)

${ }^{30}$ S. Ahn, F. Ren, E. Patrick, M. E. Law, S. J. Pearton, and A. Kuramata, "Deuterium incorporation and diffusivity in plasmaexposed bulk $\mathrm{Ga}_{2} \mathrm{O}_{3}$," Applied Physics Letters 109, 242108 (2016) https://doi.org/10.1063/1.4972265

${ }^{31}$ S. Ahn, F. R., E. Patrick, M. E. Law, S. J. Pearton, and A. Kuramata, "Thermal stability of implanted or plasma exposed deuterium in single crystal $\mathrm{Ga}_{2} \mathrm{O}_{3}$," ECS Journal of Solid State Science and Technology 6, Q3026-
Q3029 (2017).

${ }^{32}$ V. M. Reinertsen, P. M. Weiser, Y. K. Frodason, M. E. Bathen, L. Vines, and K. M. Johansen, "Anisotropic and trap-limited diffusion of hydrogen/deuterium in monoclinic gallium oxide single crystals," Applied Physics Letters 117 (2020), 10.1063/5.0027333. https://doi.org/10.1063/5.0027333

${ }^{33}$ M. Stavola, Identification of Defects in Semiconductors, Vol. $51 \mathrm{~B}$ in the series "Semiconductors and Semimetals" (Academic Press, 1999).

${ }^{34}$ A. Kyrtsos, M. Matsubara, and E. Bellotti, "Migration mechanisms and diffusion barriers of vacancies in $\mathrm{Ga}_{2} \mathrm{O}_{3}$,' Phys. Rev. B 95, 245202 (2017)

${ }^{35}$ W. B. Fowler, M. Stavola, Y. Qin, and P. Weiser, "Trapping of multiple $\mathrm{H}$ atoms at the $\mathrm{Ga}(1)$ vacancy in $\beta-\mathrm{Ga}_{2} \mathrm{O}_{3}$," Applied Physics Letters 117, 142101 (2020) https://doi.org/10.1063/5.0024269

${ }^{56}$ I. Makkonen, E. Korhonen, V. Prozheeva, and F. Tuomisto, "Identification of vacancy defect complexes in transparent semiconducting oxides $\mathrm{ZnO}$, $\mathrm{In}_{2} \mathrm{O}_{3}$ and $\mathrm{SnO}_{2}$," J. Phys.: Condens. Matter 28, 224002 (2016).

${ }^{37}$ J. F. Ziegler and J. P. Biersack, "SRIM-2013 software package," see http://www.srim.org (2013).

${ }^{38}$ M. J. Puska, C. Corbel, and R. M. Nieminen, "Positron trapping in semiconductors,' Phys. Rev. B 41, 9980-9993 (1990)

${ }^{39}$ M. D. McCluskey, "Point defects in $\mathrm{Ga}_{2} \mathrm{O}_{3}$," Journal of Applied Physics 127, 101101 (2020) https://doi.org/10.1063/1.5142195

${ }^{40}$ Z. Galazka, K. Irmscher, R. Schewski, I. M. Hanke, M. Pietsch, S. Ganschow, D. Klimm, A. Dittmar, A. Fiedler, T. Schroeder, and M. Bickermann, "Czochralski-grown bulk $\beta-\mathrm{Ga}_{2} \mathrm{O}_{3}$ single crystals doped with mono, di-, tri-, and tetravalent ions," Journal of Crystal Growth 529, 125297 (2020)

${ }^{41}$ A. Kuramata, K. Koshi, S. Watanabe, Y. Yamaoka, T. Masui, and S. Yamakoshi, "High-quality $\beta-\mathrm{Ga}_{2} \mathrm{O}_{3}$ single crystals grown by edge-defined film-fed growth," Japanese Journal of Applied Physics 55, 1202A2 (2016)

${ }^{42}$ T. Zacherle, P. C. Schmidt, and M. Martin, "Ab initio calculations on the defect structure of $\beta$ - $\mathrm{Ga}_{2} \mathrm{O}_{3}$," Phys. Rev. B 87, 235206 (2013)

${ }^{43}$ P. Deák, Q. Duy Ho, F. Seemann, B. Aradi, M. Lorke, and T. Frauenheim, "Choosing the correct hybrid for defect calculations: A case study on intrinsic carrier trapping in $\beta-\mathrm{Ga}_{2} \mathrm{O}_{3}$," Phys. Rev. B 95, 075208 (2017)

${ }^{44}$ J. B. Varley, J. R. Weber, A. Janotti, and C. G. Van de Walle, "Oxygen vacancies and donor impurities in $\beta$ - $\mathrm{Ga}_{2} \mathrm{O}_{3}$," Applied Physics Letters 97, 142106 (2010) https://doi.org/10.1063/1.3499306 


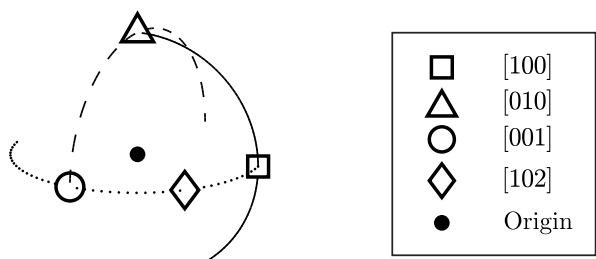




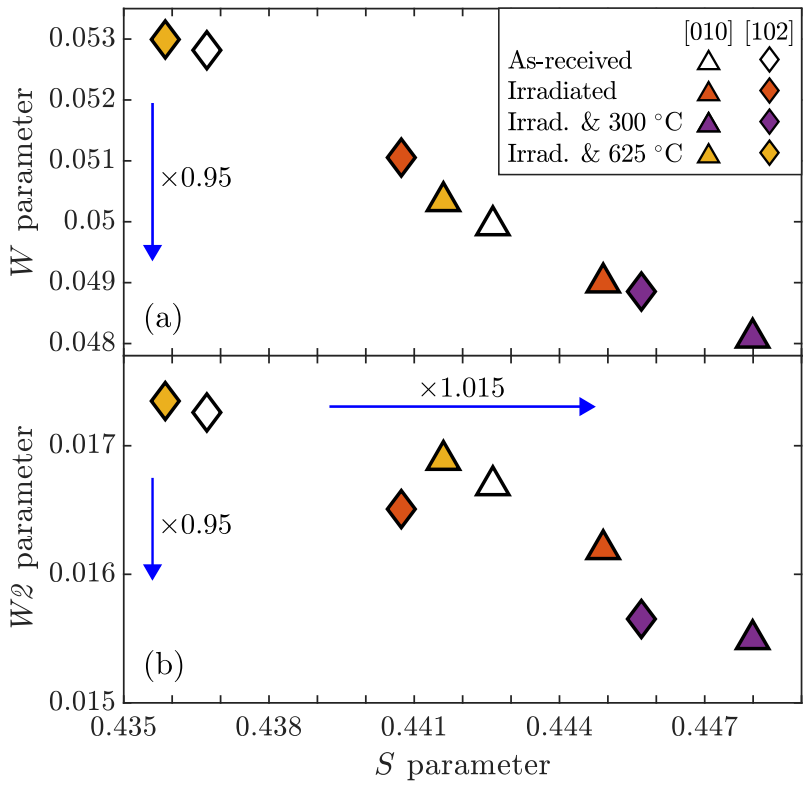




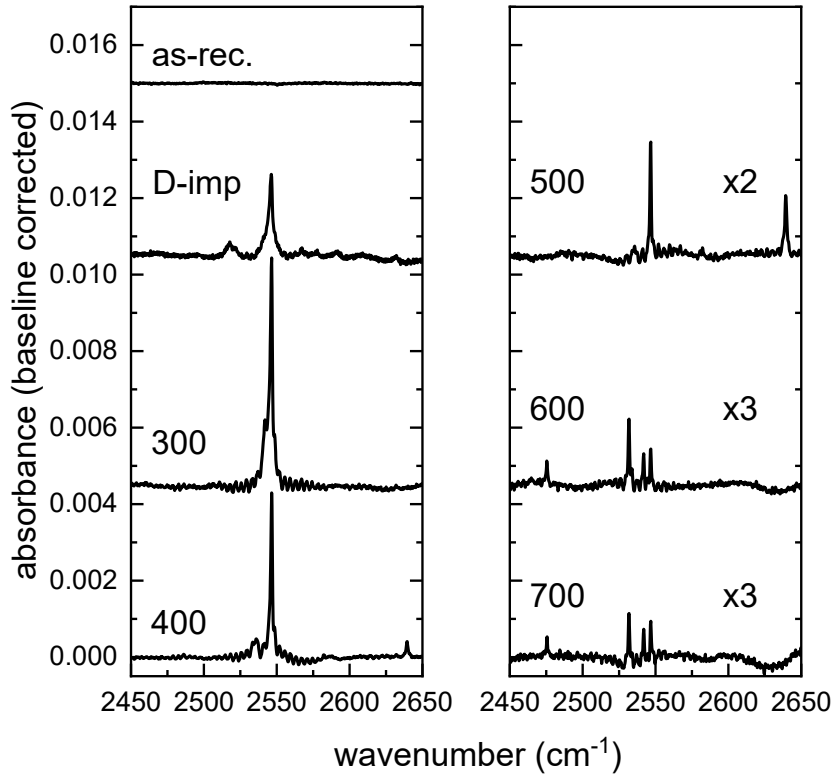




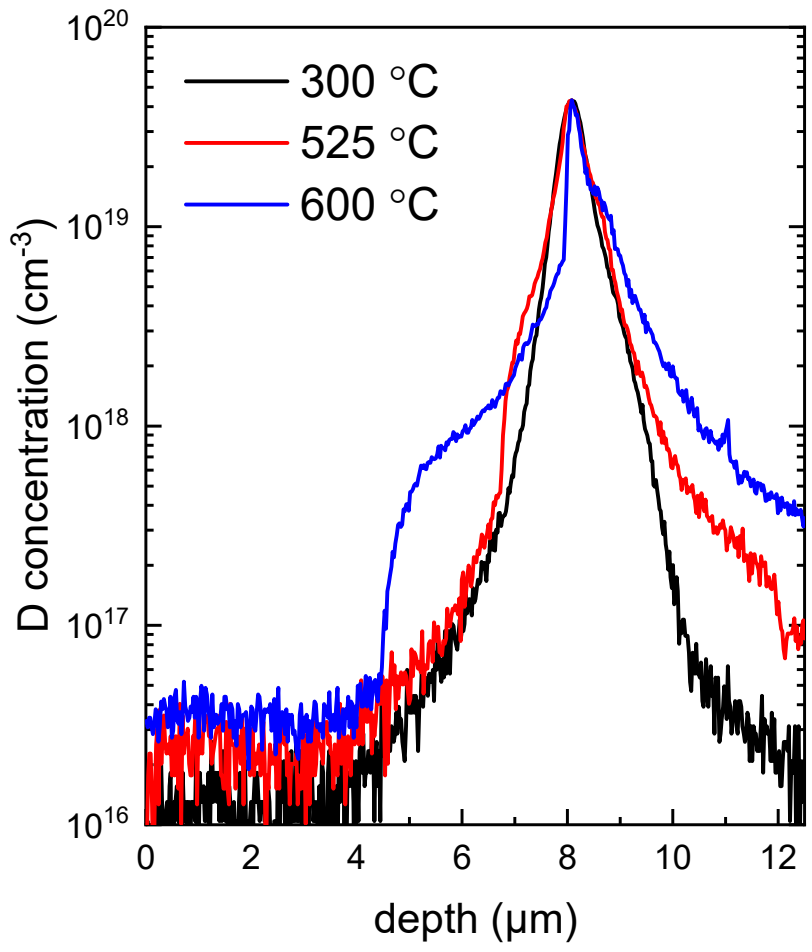

